\title{
Store-Operated Calcium Channels Contribute to Remifentanil-Induced Postoperative Hyperalgesia via Phosphorylation of CaMKIl $\alpha$ in Rats
}

\author{
Zhenhui Zhou $\mathbb{D}^{1, *}$ \\ Meng $\mathrm{MaO}^{2}$,* \\ Xuechun Cai (iD) \\ Wei Zhu (D) ${ }^{\prime}$ \\ Jie $\operatorname{Sun}^{3}$
}

'Department of Anesthesiology and Perioperative Medicine, The First Affiliated Hospital of Nanjing Medical University, Nanjing, Jiangsu, People's Republic of China; ${ }^{2}$ Department of Anesthesiology, The Affiliated Stomatological Hospital of Nanjing Medical University, Nanjing, Jiangsu, People's Republic of China; ${ }^{3}$ Department of Anesthesiology, Zhongda Hospital, Medical School, Southeast University, Nanjing, Jiangsu, People's Republic of China

*These authors contributed equally to this work

Correspondence: Jie Sun

Department of Anesthesiology, Zhongda Hospital, Medical School, Southeast University, Nanjing, 210009, People's

Republic of China

Tel +8615895971012

Email dgsunjie@hotmail.com

Wei Zhu

Department of Anesthesiology and Perioperative Medicine, The First

Affiliated Hospital of Nanjing Medical

University, Nanjing, 210009, People's

Republic of China

$\mathrm{Tel}+8618905182820$

Email zhuweijsph@163.com
Purpose: The mechanisms of remifentanil-induced postoperative hyperalgesia (RIPH) remain unclear. Store-operated calcium channels (SOCCs) are mainly comprised of stromal interaction molecules 1 (STIM1) and pore-forming subunits (Orai1). They were found to take a pivotal part in $\mathrm{Ca}^{2+}$-dependent procedures and involved in the development of central sensitization and pain. $\mathrm{Ca}^{2+} /$ calmodulin-dependent protein kinase $\mathrm{II} \alpha$ (CaMKII $\alpha$ ), regulated by $\mathrm{Ca}^{2+} /$ calmodulin complex, has been shown to have a crucial role in RIPH. This study aims to determine whether SOCCs contribute to RIPH via activating CaMKII $\alpha$.

Materials and Methods: Intra-operative infusion of remifentanil $\left(1.0 \mu \mathrm{g} \mathrm{kg}^{-1} \mathrm{~min}^{-1}\right.$, $60 \mathrm{~min}$ ) was used to establish a RIPH rat model. The SOCCs blocker (YM-58483) was applied intrathecally to confirm the results. Animal behavioral tests including paw withdrawal thermal latency (PWTL) and paw withdrawal mechanical threshold (PWMT) were performed at $-24,2,6,24,48 \mathrm{~h}$ after incision and remifentanil treatments. The protein expression of STIM1, Orai1, CaMKII $\alpha$, and p-CaMKII $\alpha$ was assayed with Western blot, and the number of STIM1 and Orail positive cells was shown by immunofluorescence.

Results: Remifentanil administration significantly induced postoperative mechanical and thermal hyperalgesia, as well as increased STIM1 and Orai1 protein expression in the spinal dorsal horn. Furthermore, the intrathecal administration of YM-58483 effectively alleviated remifentanil-induced postoperative mechanical and thermal hyperalgesia according to the behavioral tests. In addition, YM-58483 suppressed the phosphorylation of CaMKII $\alpha$ but had no effect on the expression of STIM1 and Orai1.

Conclusion: Our study demonstrated that SOCCs are involved in RIPH. The over-expressed STIM1 and Orail in the spinal cord contribute to RIPH via mediating the phosphorylation of CaMKII $\alpha$. Blockade of SOCCs may provide an effective therapeutic approach for RIPH.

Keywords: remifentanil, hyperalgesia, SOCCs, CaMKII $\alpha$

\section{Introduction}

Remifentanil is a potent short-acting $\mu$-opioid receptor agonist that has unique pharmacokinetic properties with rapid metabolism, making it a common intraoperative analgesic. ${ }^{1}$ Although it is widely used in the clinic, increasing experimental and clinical researches suggested that remifentanil is associated with paradoxical nociceptive effect, known as remifentanil-induced postoperative hyperalgesia (RIPH). ${ }^{2-5}$ There have been studies investigating how remifentanil causes hyperalgesia; however, the specific mechanism remains unclear. 
Store-operated calcium channels (SOCCs) are $\mathrm{Ca}^{2+}$ selective cation channels, which are implicated in the $\mathrm{Ca}^{2+}$ influx pathway in many different cell types. The SOCCs system is comprised of stromal interaction molecules (STIM) and pore-forming subunits (Orai). The STIM is located on the surface of the endoplasmic reticulum (ER), and the Orai is located in the plasma membrane. ${ }^{6}$ Especially, STIM1 and Orai1, two key components of SOCCs, play a crucial role in mediating store-operated $\mathrm{Ca}^{2+}$ entry (SOCE) and maintaining resting calcium homeostasis. ${ }^{7}$ In neurons, $\mathrm{Ca}^{2+}$ is crucial to the development of long-term potentiation (LTP) in the synaptic plasticity, which leads to the formation and the maintenance of pain hypersensitivity. ${ }^{8}$ Accumulated evidence has been manifested that all the components of SOCCs are expressed in the central nervous system (CNS) and involved in neuronal functions and pathological status, such as Parkinson's disease (PD), ${ }^{9}$ Alzheimer's disease (AD), ${ }^{10}$ and chronic pain. ${ }^{11,12}$ In addition, SOCE is activated by the depletion of ER calcium stores and can be blocked by YM-58483, a potent SOCCs channel blocker. ${ }^{11}$ Recent studies reported that YM-58483 was able to reverse thermal and mechanical hypersensitivity in spared nerve injury (SNI) and inflammatory pain models. ${ }^{13,14}$ However, the relationship between SOCCs and RIPH remains to be elusive.

$\mathrm{Ca}^{2+} /$ calmodulin-dependent protein kinase $\mathrm{II} \alpha$ (CaMKII $\alpha$ ), a multifunctional serine/threonine kinase, was gradually revealed a vital role in forming and maintaining RIPH. ${ }^{15,16}$ CaMKII $\alpha$ is regulated by $\mathrm{Ca}^{2+} /$ calmodulin complex. Elevated intracellular $\mathrm{Ca}^{2+}$ and calmodulin levels are essential in the activation of CaMKII $\alpha .{ }^{17}$ CaMKII $\alpha$ is involved in LTP and synaptic plasticity by decoding synaptic $\mathrm{Ca}^{2+}$ oscillations. ${ }^{18,19}$ Temporarily elevated levels of p-CaMKII $\alpha$ in the CNS are associated with central sensitization and pain hypersensitivity. ${ }^{20}$ Furthermore, SOCE induced by chronic ER $\mathrm{Ca}^{2+}$ depletion may lead to increased intracytoplasmic $\mathrm{Ca}^{2+}$ concentration, resulting in CaMKII $\alpha$ activation. $^{21}$ Therefore, we hypothesized that the inhibition of SOCE could reduce the phosphorylation of CaMKII $\alpha$ in the spinal cord, thereby alleviating RIPH.

In the present study, we investigated the expression level of SOCCs in the spinal dorsal horn by establishing a rat model of RIPH. Since CaMKII $\alpha$ is activated by $\mathrm{Ca}^{2+}$ and involved in RIPH, we next explored whether SOCCs contribute to RIPH via phosphorylation of CaMKII $\alpha$. Our results provide a molecular mechanism and therapeutic approach on RIPH.

\section{Materials and Methods}

\section{Animals}

Mature male Sprague Dawley rats (2-3 months old, 360$380 \mathrm{~g}$ ) were purchased from the Zhejiang Academy of Medical Sciences. All procedures were performed following the approved guidelines of Southeast University, and all animal model experiments were tested with the approval of the Animal Experimental Ethics Committee of Southeast University (Ethical permission code: 20200310006). Rats were housed under controlled environmental conditions (room temperature of $24 \pm 1{ }^{\circ} \mathrm{C}$ and relative humidity of $55 \% \pm 10 \%$ ) with a 12 -h light/dark cycle. Five rats per cage were received standard rats chow and water ad libitum. The rats were randomly divided into four groups in each experiment, with 10 rats in each group before any assessment was performed.

\section{Surgical Procedure}

The plantar incision was performed as described in the previous article. ${ }^{22}$ A $1.0 \mathrm{~cm}$ longitudinal plantar incision through the skin and fascia of the right hind paw from the edge of the heel to the toe was made with a No. 11 blade. The plantaris muscle was separated by ophthalmic forceps and incised longitudinally, and the tendon should be protected from being severed. The skin was sutured with 4-0 sutures, and the wound site was covered with Aureomycin ointment to prevent infection. Rats in the control group received sham procedures without skin incisions.

\section{Experiment Related Procedures}

For intravenous drug administration, a 24-gauge catheter rinsed with heparinized saline $(100 \mathrm{U} / \mathrm{mL})$ was cannulated in the rat tail vein. For intrathecal drug administration, a lumbar puncture technique was used to accomplish a successful intrathecal injection. ${ }^{23}$ Sevoflurane anesthetized rats were placed in the prone position, exposing the lumbar vertebral space between L4 and L5. A $20 \mu \mathrm{L}$ microinjector was inserted vertically into the lumbar intervertebral space. Brisk tail movement indicated successful intrathecal injection.

\section{Drug Administration}

Remifentanil (Yichang Renfu Pharmaceutical Co., Yichang, China) was dissolved in $0.9 \%$ saline and injected intravenously (i.v.) for 60 minutes, the infusion rate was 1.0 
$\mu \mathrm{g}^{-1} \cdot \mathrm{kg}^{-1} \cdot \min$. Rats in the control group received the same volume of saline $\left(0.1 \mathrm{~mL} \mathrm{~kg}^{-1} \mathrm{~min}^{-1}, 60 \mathrm{~min}\right)$. YM-58483 (MedChemexpress LLC, Princeton, NJ, USA) was dissolved in PEG300 (MedChemexpress LLC, Princeton, NJ, USA) and injected intrathecally (i.th.) at the concentration of $10 \mu \mathrm{L}(1000$ $\mu \mathrm{mol} / \mathrm{L}) 2 \mathrm{~h}$ before each behavior test. The same volume of PEG300 was used as the control treatment. All drugs were administered under sevoflurane anesthesia (induction, 3.0\%; maintenance, 1.0\%; Hengrui Pharmaceutical Co., Ltd, Shanghai, China). The detailed process and grouping of the experiment are shown in Figure 1A-D.

\section{Nociceptive Behavioral Tests}

Paw withdrawal mechanical threshold (PWMT) was measured using von Frey filaments (Aesthesio, Danmic Global, LLC, Campbell, CA, USA) according to the "up-down" method. ${ }^{24}$ Briefly, each rat was placed in an individual plastic box with a metallic mesh bottom and acclimated for $30 \mathrm{~min}$. The calibrated filaments were perpendicular to the plantar surface of the hind paw for $6 \mathrm{~s}$, and the positive response was defined as a brisk paw withdrawal or flinching. Under the condition of a positive response, the next lower force filament was applied. If in a negative response, the next greater force filament was applied. The up-down algorithm was applied to calculate the $50 \%$ likelihood of the PWMT. ${ }^{25}$

Paw withdrawal thermal latency (PWTL) was measured using a thermal testing apparatus (BME-410C, Inst of Biomedical Engineering, CAMS, Tianjin, China). Each rat was placed in an individual plastic box on a glass surface and acclimated for $30 \mathrm{~min}$. The targeted hind paw was focused by a mobile radiant heat source located under the glass until the positive response occurred, and the time was recorded as the PWTL. The test was repeated three times at 10 -min intervals. The average value of the three trials was evaluated as the mean PWTL. To protect from potential tissue damage, a 30 s cut-off was set. ${ }^{25}$ The observer of behavioral tests was blinded to the treatment.

PWMT and PWTL were measured at 24 hours before remifentanil administration as baseline and then at 2, 6, 24, and 48 hours after remifentanil administration.

\section{Western Blot}

After the last behavioral test at $48 \mathrm{~h}$, rats were deeply anesthetized with sevoflurane and spinal cord tissues were removed from the enlarged lumbar region (L4-L6) for Western blotting analysis. Spinal cord tissues were extracted in RIPA buffer (Beyotime Biotech Inc., Shanghai, China) containing protease and phosphatase inhibitors. After centrifuging $(\times 12,000 \mathrm{rpm})$ at $4^{\circ} \mathrm{C}$ for $20 \mathrm{~min}$, the supernatant fluid of each sample was obtained and protein concentration was detected by BCA protein assay. Twenty-four micrograms of each protein sample was electrophoresis with $10 \%$ SDS-PAGE, then transferred to PVDF membrane (Millipore Corp, Billerica, MA, USA). The membrane was blocked with $5 \%$ bovine serum albumin for $1 \mathrm{~h}$ at room temperature and then incubated with the following primary antibodies: rabbit monoclonal anti-STIM1 (\#5668,1:1000, CST, Danvers, MA, USA), mouse monoclonal anti-Orail (ab175040, $5 \mu \mathrm{g} / \mu \mathrm{L}$, Abcam, Cambridge, UK), rabbit monoclonal anti-phospho-CaMKII (Thr286) (\#12716,1:1000, CST, Danvers, MA, USA), mouse monoclonal antiCaMKII $\alpha$ (6G9) (\#50049,1:1000, CST, Danvers, MA, USA) and rabbit anti-GADPH (\#10494-1-AP, 1:20000, Proteintech, Wuhan, China) overnight at $4^{\circ} \mathrm{C}$. The biotinylated secondary antibody (anti-rabbit IgG (GB23303,1:3000, Servicebio Technology Co., Ltd, Wuhan, China) or antimouse IgG (GB23301,1:3000, Servicebio Technology Co., Ltd, Wuhan, China)) was used to incubate the membranes for $1 \mathrm{~h}$ at room temperature. After rinsing in TBST and incubated in ECL liquid ((Beyotime Biotech Inc., Shanghai, China), target protein bands were detected using a ChemiScope6100 imaging system (Clinx Science Instruments, Co., Ltd, Shanghai, China) and quantified by the Image J software (National Institute of Health, Bethesda, MD, USA).

\section{Immunofluorescence}

After the last behavioral test at $48 \mathrm{~h}$, rats were deeply anesthetized with sevoflurane and instilled with 0.1 $\mathrm{M}$ phosphate buffer saline (PBS) and 4\% paraformaldehyde (PFA). The L4-L6 spinal cords were rapidly removed, post-fixed in 4\% PFA for $24 \mathrm{~h}$ and dehydrated in $30 \%$ sucrose at $4{ }^{\circ} \mathrm{C}$ for $72 \mathrm{~h}$. Spinal cord tissues were embedded with O.C.T. Compound (SAKURA Tissue-Tek ${ }^{\circledR}$ O.C.T. Compound, Sakura Finetek, USA) and cut into 30$\mu \mathrm{m}$-thick sections using a freezing microtome. Tissue sections were permeabilized in PBS containing $0.1 \%$ Triton $\mathrm{X}-100$ for $2 \mathrm{~h}$ after being rinsed in PBS and then blocked in $10 \%$ bovine serum albumin for $1 \mathrm{~h}$. Sections were then incubated with the mixed primary antibodies (rabbit antiSTIM1, \#5668, 1:200, CST, Danvers, MA, USA, Orai1; mouse anti-Orail ab175040, $1 \mu \mathrm{g} / \mu \mathrm{L}$, Abcam, Cambridge, $\mathrm{UK}$ ) for $48 \mathrm{~h}$ at $4{ }^{\circ} \mathrm{C}$. Subsequently, sections were washed in PBS and incubated with the blended secondary antibodies (Alexa Fluor 488 conjugated to goat anti-mouse IgG, 1:500, Proteintech, Wuhan, China; Alexa Fluor 532 conjugated to goat anti-rabbit $\mathrm{IgG}, 5 \mu \mathrm{g} / \mathrm{mL}$, Invitrogen, 


\section{Experiment 1}

A Behavior Testing

Behavior Testing

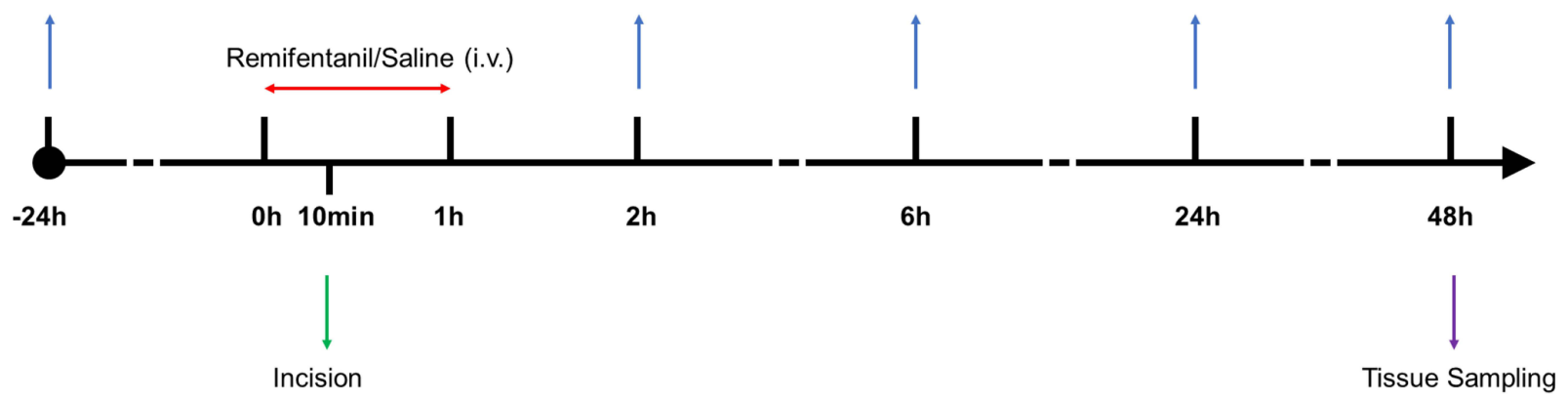

B

\begin{tabular}{|c|c|}
\hline Groups $(\mathbf{n = 1 0})$ & Treatments \\
\hline $\mathrm{NS}$ & Saline $0.1 \mathrm{ml} \cdot \mathrm{kg}^{-1} \cdot \mathrm{min}^{-1}(60 \mathrm{~min}$, i.v. $)$, without incision \\
\hline Inci & Saline $0.1 \mathrm{ml} \cdot \mathrm{kg}^{-1} \cdot \mathrm{min}^{-1}(60 \mathrm{~min}$, i.v. $)+$ incision \\
\hline Remi & Remifentanil $1.0 \mu \mathrm{gg} \cdot \mathrm{kg}^{-1} \cdot \mathrm{min}^{-1}(60 \mathrm{~min}$, i.v. $)$, without incision \\
\hline Inci+Remi & Remifentanil $1.0 \mu \mathrm{gg} \cdot \mathrm{kg}^{-1} \cdot \mathrm{min}^{-1}(60 \mathrm{~min}$, iv. $)+$ incision \\
\hline
\end{tabular}

\section{Experiment 2}

C

Behavior Testing

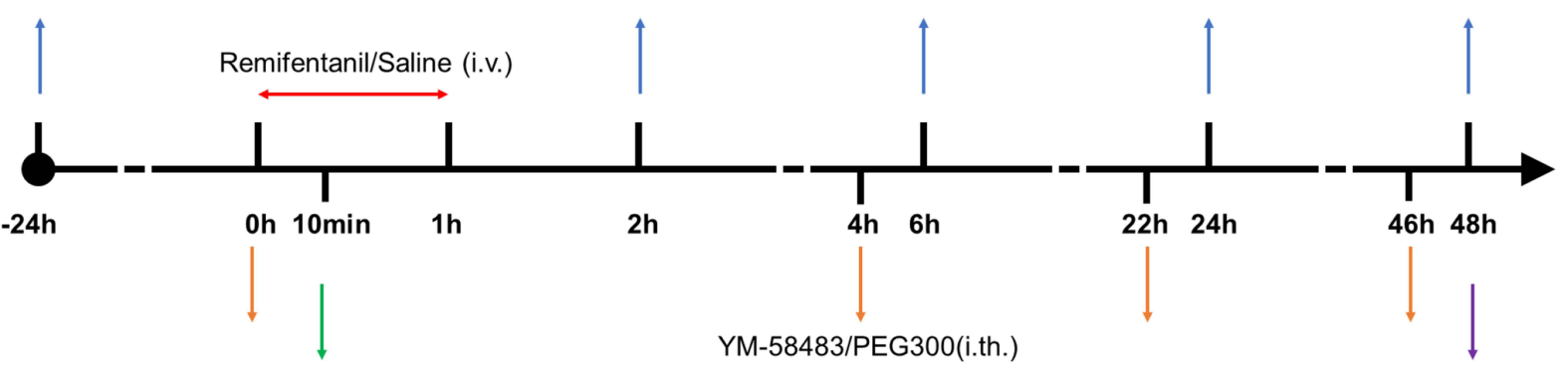

Incision

Tissue Sampling

D

\begin{tabular}{c} 
Groups(n=10) \\
\hline NS+Vehicle \\
Inci+Vehicle \\
Inci+Remi+Vehicle \\
Inci+Remi+YM-58483
\end{tabular}

\section{Treatments}

Saline $0.1 \mathrm{ml} \cdot \mathrm{kg}^{-1} \cdot \mathrm{min}^{-1}(60 \mathrm{~min}$, i.v.), without incision+PEG300 $10 \mu \mathrm{l}$, i.th.

Saline $0.1 \mathrm{ml} \cdot \mathrm{kg}^{-1} \cdot \mathrm{min}^{-1}(60 \mathrm{~min}$, i.v. $)+$ incision+PEG300 $10 \mu \mathrm{l}$, i.th.

Remifentanil $1.0 \mu \mathrm{g} \cdot \mathrm{kg}^{-1} \cdot \mathrm{min}^{-1}(60 \mathrm{~min}$, iv. $)+$ incision+PEG300 $10 \mu \mathrm{l}$, i.th.

Remifentanil $1.0 \mu \mathrm{g} \cdot \mathrm{kg}^{-1} \cdot \mathrm{min}^{-1}(60 \mathrm{~min}, \mathrm{iv})+$. incision $+\mathrm{YM}-5848310 \mathrm{nmol}$, i.th.

Figure I Detailed process and grouping of the experiment.

Notes: (A and B) Experiment I is to establish a rat RIPH model and investigate whether SOCCs are involved in RIPH. (C and D) Experiment 2 is to examine the effect of YM-58483 on RIPH and further confirm the mechanism of SOCCs involvement in RIPH.

Abbreviations: RIPH, remifentanil-induced postoperative hyperalgesia; SOCCs, store-operated calcium channels.

Carlsbad, CA, USA) for $24 \mathrm{~h}$. Images were acquired with a fluorescence microscope (MF31, Mshot, Guangzhou, China). Every slide was captured three selected fields under $10 \times$ magnification and counted the number of positive cells.

\section{Statistical Analysis}

Data were shown as means \pm SEM. Two-way analysis of variance (ANOVA) followed by Dunnett's post hoc test was applied to analyze the behavioral data. One-way ANOVA followed by Tukey's post hoc test was applied to analyze the 
Western blot and immunohistochemistry data. All statistical analyses were performed using GraphPad Prism version 8.3 (GraphPad Software Inc., San Diego, CA, USA). P $<0.05$ was considered to be statistically significant.

\section{Result}

\section{Remifentanil-Induced Mechanical and Thermal Hyperalgesia in Rats}

As shown in Figure 2A and B, there were no significant differences in basic values of PWMT and PWTL among all groups $(\mathrm{P}>0.05)$. Compared with baseline, $\mathrm{PWMT}$ and PWTL decreased in Inci, Remi and Inci + Remi groups $(\mathrm{P}<0.05)$ at different time points, and they did not show significant changes in NS group $(\mathrm{P}>0.05)$. Compared with NS group, PWMT decreased from 2 to $24 \mathrm{~h}$ and PWTL decreased from 2 to $48 \mathrm{~h}$ in Remi group $(\mathrm{P}<0.05)$. Furthermore, compared with Inci group, PWMT decreased from 6 to $48 \mathrm{~h}$ and PWTL decreased from 2 to $48 \mathrm{~h}$ in Inci + Remi group $(\mathrm{P}<0.05)$. These results indicated that the RIPH model was successfully established.

\section{The Expression of STIMI and Orail in the Spinal Dorsal Horn Increased After Remifentanil Administration}

To determine the effect of remifentanil on STIM1 and Orai1 expression in the spinal cord, we detected the SOCCs expression at $48 \mathrm{~h}$ after the administration of remifentanil. The Western blot analysis of homogenized spinal tissues showed STIM1 and Orail levels were significantly increased in Inci (STIM1: $\mathrm{P}<0.05$; Orai1: $\mathrm{P}<0.01$ ), Remi (STIM1: $\mathrm{P}<0.01$; Orail: $\mathrm{P}<0.001$ ), and Inci + Remi (STIM1:
$\mathrm{P}<0.001$; Orai1: $\mathrm{P}<0.001)$ groups as compared with NS group (Figure 3A-C). Compared with Inci group, the expression of STIM1 and Orail were increased in Inci + Remil group (STIM1: $\mathrm{P}<0.001$; Orai1: $\mathrm{P}<0.001$ ) (Figure $3 \mathrm{~A}-\mathrm{C}$ ).

Since previous studies showed that STIM1 and Orai1 are mainly expressed in the spinal dorsal horn neurons, and the decreased concentration of ER $\mathrm{Ca}^{2+}$ stores increases STIM1 and Orail puncta formation in vitro. ${ }^{11,12}$ Thus, we detected the number of STIM1 and Orai1 in the spinal dorsal horn. As shown in Figure 3D-F, STIM1 and Orail collocated in the same spinal cord cells. Consistent with the Western blot results above, remifentanil administration significantly increased the number of STIM1 and Orail positive cells $(\mathrm{P}<0.001)$.

\section{Blocking of SOCCs Reversed RIPH}

To further verify the effect of SOCCs on RIPH, a specific blocker of the SOCCs, YM-58483 was intrathecally injected into rats $2 \mathrm{~h}$ before each behavior testing. As shown in Figure 4A and B, compared with Inci + Remi + Vehicle group, administration of YM-58483 (10 nmol) with incision and remifentanil significantly increased PWMT and PWTL $(\mathrm{P}<0.05)$. Interestingly, we did not detect a difference between Inci + Vehicle group and Inci + Remi+YM-58483 group $(\mathrm{P}>0.05)$.

\section{YM-58483 Decreased Remifentanil- Induced Phosphorylation of CaMKIla in the Spinal Cord}

Next, Western blot data demonstrated that intrathecal treatment with SOCCs blocker, YM-58483, did not affect the

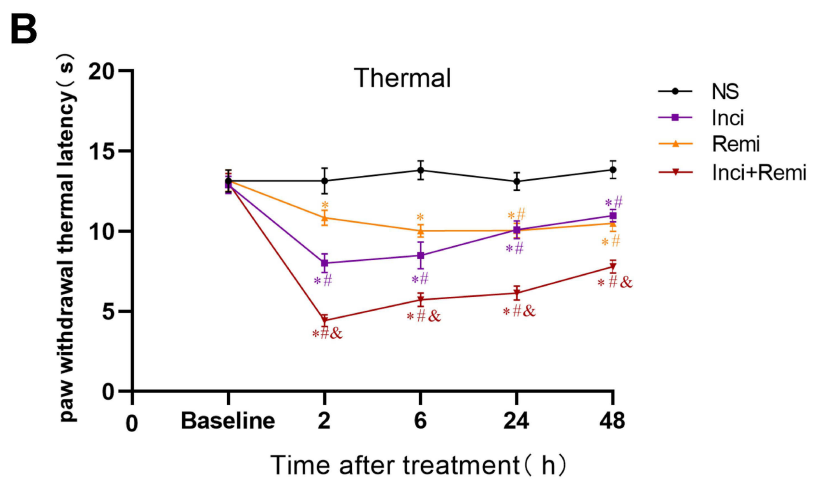

Figure 2 The effect of RIPH.

Notes: The basic values of PWMT (A) and PWTL (B) were similar in all groups. Both incision and remifentanil significantly decreased PWMT (A) and PWTL (B) compared with group NS. Furthermore, remifentanil infusion significantly increased incision-induced thermal and mechanical hyperalgesia. All the data are expressed as means \pm SEM ( $=10$ ) and analyzed by two-way ANOVA with Dunnett post hoc comparisons. ${ }^{*} \mathrm{P}<0.05$ vs baseline; ${ }^{\#} \mathrm{P}<0.05$ vs group NS; ${ }^{\text {\& }} \mathrm{P}<0.05$ vs group Inci.

Abbreviations: PWTL, paw withdrawal thermal latency; PWMT, paw withdrawal mechanical threshold; SEM, standard error of measurement; ANOVA, analysis of variance; h, hours. 
A

STIM1

GAPDH

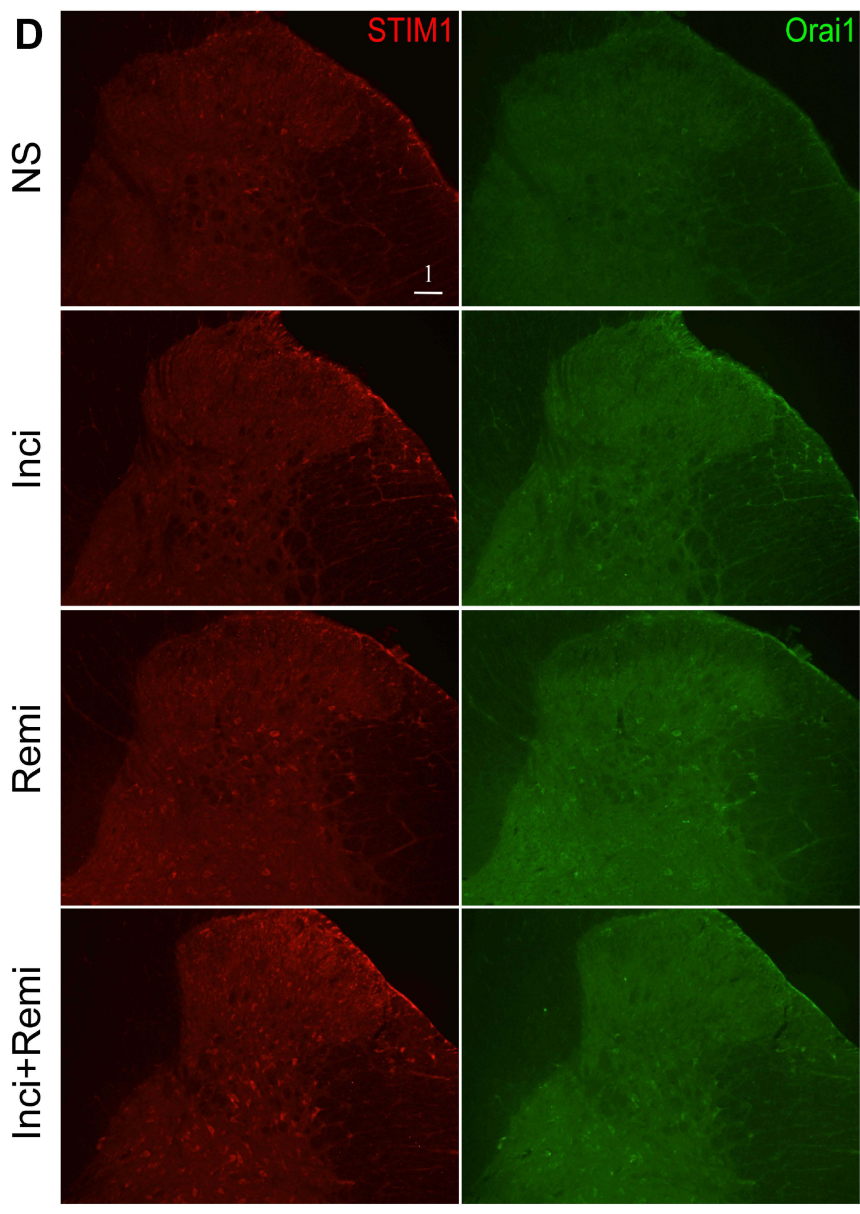

E

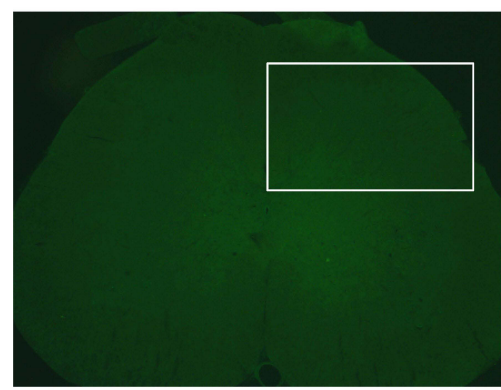

B

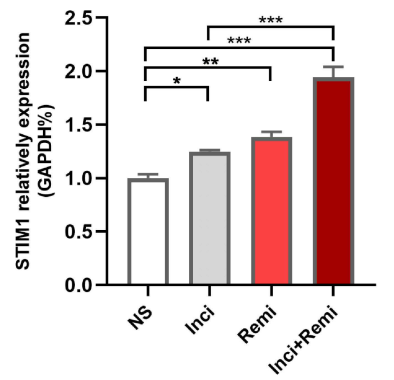

C
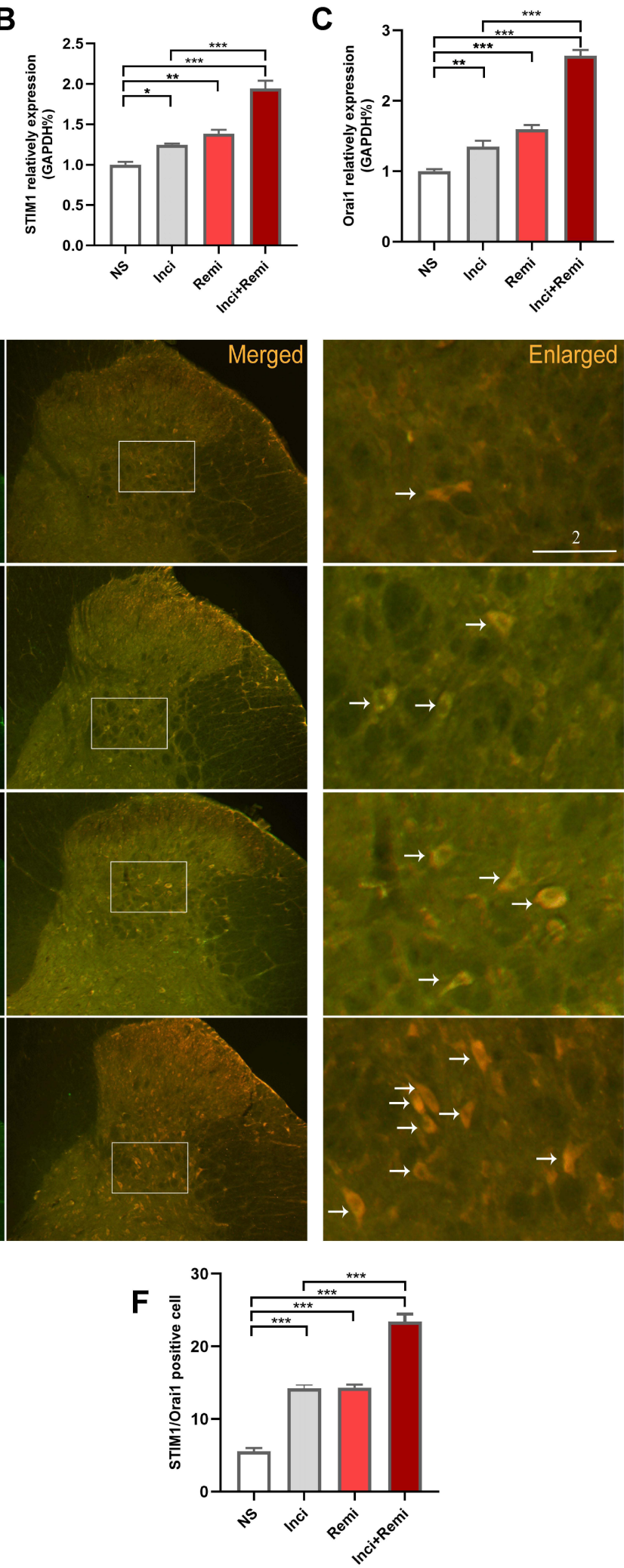

Figure 3 Expression of STIMI and Orail in rat spinal dorsal horn after RIPH.

Notes: Representative blot images (A) and statistical analysis of STIMI and Orail protein expression (B and $\mathbf{C})$ in the spinal cord ( $\mathrm{n}=4)$. The amount of STIMI and Orail proteins ( 85 and $48 \mathrm{kDa}$ ) was normalized to that of GAPDH $(36 \mathrm{kDa})$ in the same sample, and the mean STIMI and Orail level in control rats were considered to be I. Representative double immunofluorescence labeling images (D) and statistical analysis of positive cells (F) of STIMI and Orail in the spinal dorsal horn (E). Three selected fields in 3 serial sections were analyzed under a fluorescence microscope $(\times 10 ; n=3)$. Scale bar: both I and 2 are $100 \mu \mathrm{m}$. Data are expressed as means $\pm \mathrm{SEM}$ and analyzed by one-way ANOVA. $* \mathrm{P}<0.05$, $* * \mathrm{P}<0.0 \mathrm{I}$, $* * * \mathrm{P}<0.00 \mathrm{I}$. Abbreviations: STIMI, stromal interaction molecules I; GAPDH, glyceraldehyde-3-phosphate dehydrogenase. 

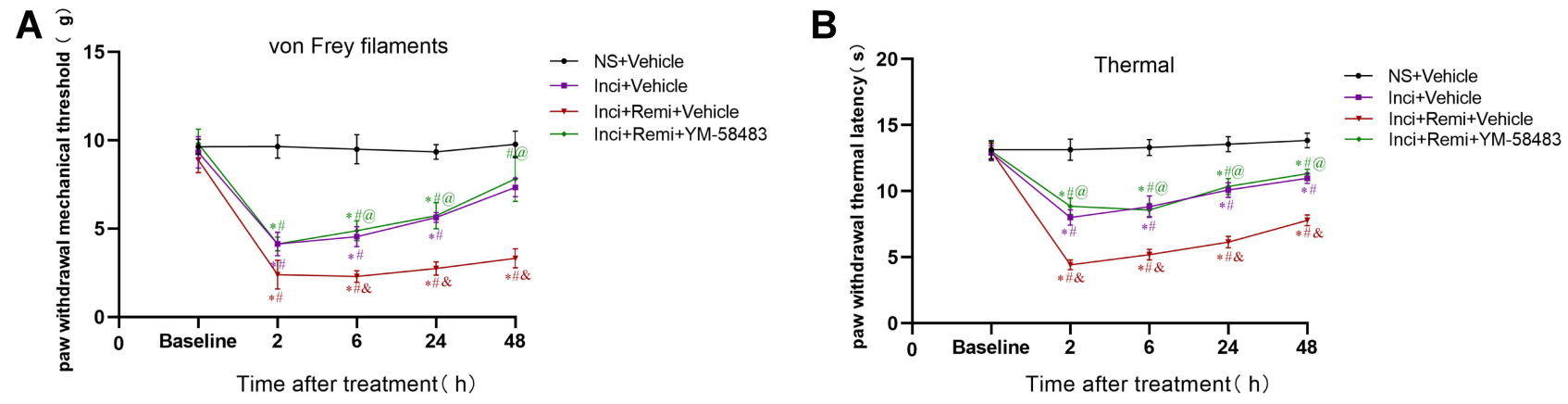

Figure 4 The effect of YM-58483 on RIPH.

Notes: Intrathecal injection of YM-58483 attenuated remifentanil-induced postoperative mechanical (A) and thermal (B) hyperalgesia. There was no difference between group Inci + Vehicle and group Inci + Remi + Vehicle. All the data are expressed as means \pm SEM ( $=10)$ and analyzed by two-way ANOVA with Dunnett post hoc comparisons. $* \mathrm{P}<0.05$ vs baseline; ${ }^{\#} \mathrm{P}<0.05$ vs group NS + Vehicle; ${ }^{\circledR} \mathrm{P}<0.05$ vs group Inci + Vehicle; ${ }^{@} \mathrm{P}<0.05$ vs group Inci + Remi + Vehicle.

Abbreviation: h, hours.

expression of STIM1 and Orail in RIPH $(\mathrm{P}>0.05)$ (Figure 5A-C). Studies have manifested that the phosphorylation of CaMKII $\alpha$ plays a crucial role in RIPH. ${ }^{20}$ Thus, we further determined whether the phosphorylation of CaMKII $\alpha$ were involved in RIPH via increased $\mathrm{Ca}^{2+}$ induced by SOCE. Compared NS + vehicle group, the expression of $\mathrm{p}$-CaMKII $\alpha$ was significantly increased in Inci + vehicle group $(\mathrm{P}<0.01)$ and Inci + Remi + vehicle group $(\mathrm{P}<0.001)$ (Figure 5D and $\mathrm{E})$. Moreover, YM-58483 suppressed the level of $\mathrm{p}$-CaMKII $\alpha$ compared with the Inci + Remi + vehicle group $(\mathrm{P}<0.01)$ (Figure $5 \mathrm{D}$ and $\mathrm{E}$ ). In addition, the protein level of CaMKII $\alpha$ did not demonstrate a significant difference among all groups $(\mathrm{P}>0.05)$ (Figure 5D and F).

\section{Discussion}

Our results suggested that SOCCs play a crucial role in RIPH. The increase of the STIM1 and Orail protein was seen in remifentanil used rats' spinal dorsal horn. Furthermore, remifentanil infusion downregulated the nociceptive thresholds (PWMT and PWTL) at different time points, which could be partly improved by YM58483 , a potent SOCCs channel blocker. The expression of p-CaMKII $\alpha$ was partly reversed by YM-58483. It is indicated that blockade of SOCCs could regulate the expression of $\mathrm{p}$-CaMKII $\alpha$ to prevent the RIPH.

Hyperalgesia refers to increased pain from a stimulus that usually provokes pain, including a reduced pain threshold and an increased response to subnormal stimuli. $^{26}$ Opioid-induced hyperalgesia $(\mathrm{OIH})$ is known as a paradoxical increase in sensitivity to painful stimuli associated with opioid therapy, resulting in slower patient's recovery after surgery and more consumption of analgesics. ${ }^{27,28}$ As a kind of opioid, remifentanil is widely used in general anesthesia due to its unique properties. Indeed, remifentanil has high lipid solubility, allowing for a rapid onset of effect; It is rapidly metabolized by non-specific plasma and tissue esterases and hence a rapid recovery. ${ }^{1}$ Although remifentanil offers significant advantages in the clinic, compared with other opioids, it has the highest reported incidence of hyperalgesia, known as remifentanil-induced hyperalgesia $(\mathrm{RIH}) .{ }^{29}$ With regard to the postoperative period, RIH results in increased opioid consumption and pain sensibility, which causes discomfort in patients and longer stay in hospital than expected.

Evidence from both human and animal models has reported that RIH is caused by exposure to a high dosage of remifentanil. ${ }^{30}$ Clinical trials are difficult to draw conclusions about the incidence of RIH due to heterogeneity of remifentanil infusion regimens, maintenance of anesthesia, duration of infusion, cumulative dose of remifentanil, and pain measures. However, it appears that intraoperative remifentanil infusion rates greater than $0.2 \mu \mathrm{g}^{-1} \cdot \mathrm{kg}^{-1} \cdot \mathrm{min}$ is associated with lower pain thresholds, which suggests hyperalgesia. ${ }^{1}$ On the other hand, animal researches showed that $1.0 \mu \mathrm{g}^{-1} \cdot \mathrm{kg}^{-1} \cdot \min$ for 60 min could induce hyperalgesia in rats. Therefore, the dosage of remifentanil $\left(1.0 \mu \mathrm{g}^{-1} \cdot \mathrm{kg}^{-1} \cdot \mathrm{min}, 60 \mathrm{~min}\right)$ was chosen to establish a rat model of hyperalgesia, which approximates the equivalent dose conversed between humans and rats. ${ }^{31}$ Furthermore, 


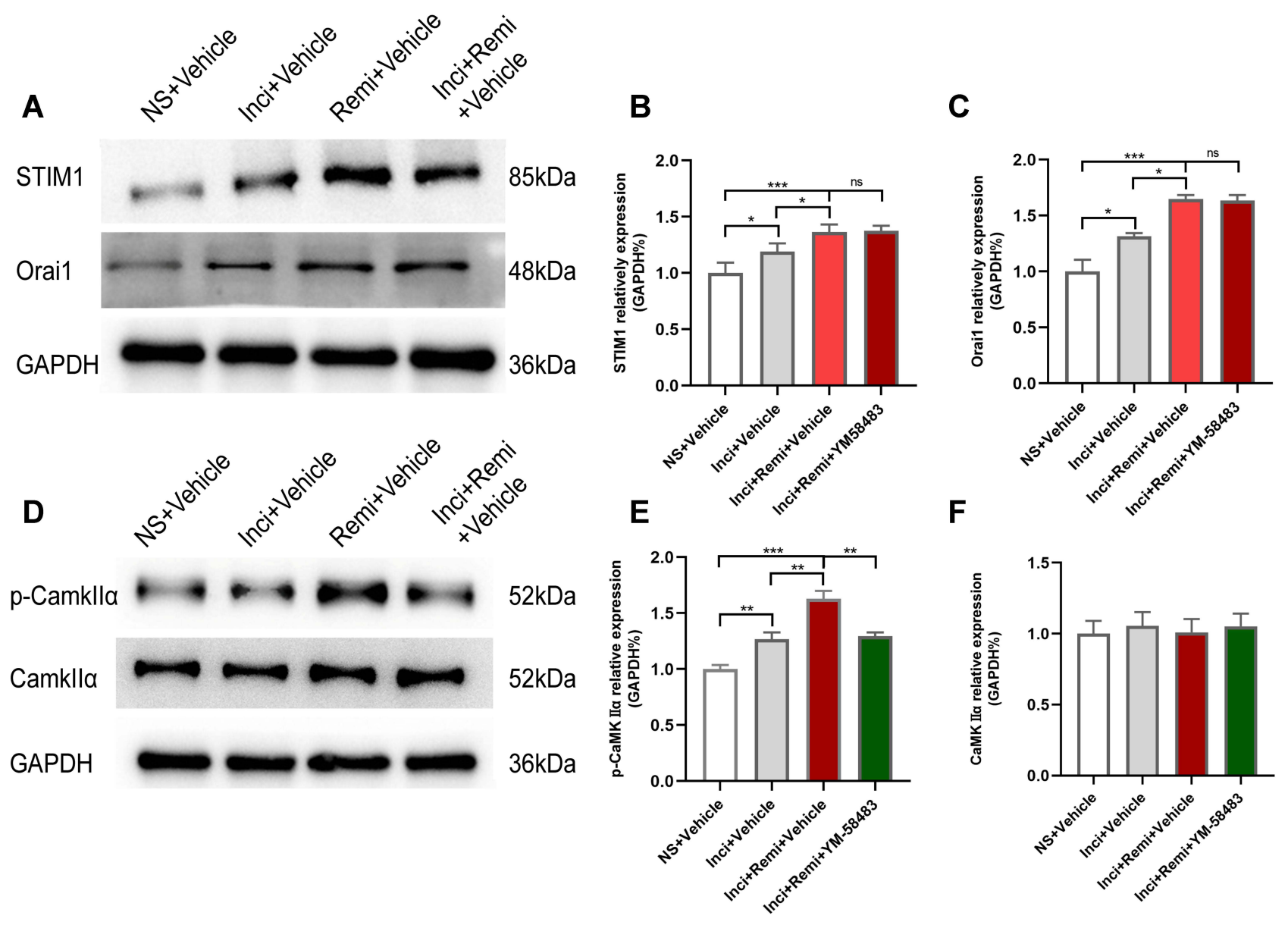

Figure 5 The effects of YM-58483 on SOCCs and p-CaMKIl $\alpha$ after RIPH.

Notes: Representative blot images (A) and statistical analysis of STIMI and Orail protein expression (B and $\mathbf{C}$ ) in the spinal cord ( $\mathrm{n}=4)$. The amount of STIMI and Orail proteins $(85$ and $48 \mathrm{kDa})$ was normalized to that of GAPDH $(36 \mathrm{kDa})$ in the same sample, and the mean STIMI and Orail level in control rats were considered to be I. Representative blot images (D) and quantification (E and F) of p-CaMKIl $\alpha$ and CaMKIl $\alpha$ protein levels in the spinal cord ( $n=4)$. The amount of $\mathrm{p}-\mathrm{CaMKIl} \alpha$ and CaMKIl $\alpha$ proteins $(52 \mathrm{kDa})$ was normalized to that of GAPDH (36 kDa) in the same sample, and the mean P-CaMKIl $\alpha$ and CaMKIl $\alpha$ level in control rats was considered to be I. Data are expressed as means \pm SEM and analyzed by one-way ANOVA and person correlation analysis. *P $<0.05, * * \mathrm{P}<0.0 \mathrm{I}$, ***P $<0.00 \mathrm{I}$.

Abbreviation: $\mathrm{CaMKIl \alpha}, \mathrm{Ca}^{2+} /$ calmodulin-dependent protein kinase Il $\alpha$.

remifentanil anesthesia during operation is more common in clinical practice; thus, we investigated the effects of incision plus remifentanil on pain sensitivity. Interestingly, intra-operative exposure to remifentanil apparently increased the incision-induced mechanical and thermal hyperalgesia.

SOCCs are activated by the release or depletion of $\mathrm{Ca}^{2}$ ${ }^{+}$from the ER, influencing neurotransmitter release and synaptic plasticity. ${ }^{32,33}$ SOCE is a major mechanism for triggering $\mathrm{Ca}^{2+}$ enter into cells, which is required for many $\mathrm{Ca}^{2+}$-dependent cellular functions, such as enzymatic activity. There are two key components of SOCCs-stromal interaction molecule 1 (STIM1) and Orai1. ${ }^{34}$ Growing evidence manifests that SOCCs play a crucial role in pain disorders, such as SNI, inflammation, and acute pain. ${ }^{13,35,36}$ Our results indicated that continuous remifentanil infusion increases the expression level of STIM1 and Orail in the spinal dorsal horn, which has a vital effect on maintained remifentanil-induced thermal and mechanical hypersensitivities. Furthermore, YM58483 , a potent SOCCs channel blocker, was reported to have analgesic actions in both acute and chronic pain. ${ }^{13}$ Our behavior testing results confirmed that intrathecal administration of YM-58483 reversed thermal and mechanical hyperalgesia, suggesting an action of SOCCs in regulating RIPH.

However, we further tested the protein expression of STIM1 and Orai1 by Western blot and found that YM58483 could not decrease their levels during the development of RIPH. It is well documented that a decrease in ER $\mathrm{Ca}^{2+}$ leads to the intracellular redistribution of STIM1 at the ER membrane. The STIM1 puncta activates the Orail 
channel at the plasma membrane, allowing $\mathrm{Ca}^{2+}$ entry into the cellular. ${ }^{12}$ However, the core mechanisms of action remain to be determined. Previous studies have manifested that YM-58483 blocks SOCE and dose-dependently inhibits the CaMKII $\alpha$ activation in arthritic pain models. ${ }^{14}$ Our results showed that the analgesic effect of YM-58483 in RIPH is not through impacting the expression of SOCCs. Thus, we further detected the protein expression of $\mathrm{CaMKII} \alpha$ and $\mathrm{p}-\mathrm{CaMKII} \alpha$.

CaMKII $\alpha$, as a major CaMKII isoform expressed in the $\mathrm{CNS}$, is an essential cellular mechanism leading to and maintaining OIH. ${ }^{37}$ CaMKII $\alpha$ is activated by increased intracellular $\mathrm{Ca}^{2+}$. Phosphorylated CaMKII $\alpha$ is a part of LTP hypersensitivity signaling, which is involved in the sensitization of homosynapses leading to an enhanced strength of the synapse and its signal transduction. Indeed, cumulative studies confirmed that inhibiting the activation of CaMKII $\alpha$ attenuates RIPH. ${ }^{38,39}$ Furthermore, SOCCs have been shown implicated in CaMKII $\alpha$ activation and YM-58483 effectively inhibits the CaMKII $\alpha$ activation in the spinal cord in different pain models. ${ }^{14,40}$ Our results indicated that CaMKII $\alpha$ becomes activated after the intra-operative infusion of remifentanil and that this increased phosphorylation of CaMKII $\alpha$ was significantly suppressed by YM-58483.

Together, these results above indicated that exposure to remifentanil up-regulates STIM1 and Orail protein expression, further enhancing the SOCE. Increased intracellular $\mathrm{Ca}^{2+}$ leads to the phosphorylation of CaMKII $\alpha$, which is part of LTP hypersensitivity signaling contributing to RIPH (Figure 6).

\section{Conclusion}

In summary, our study demonstrated that SOCCs in rat spinal dorsal horn contribute to the development of RIPH via activation of CaMKII $\alpha$. The pharmacologic intervention of SOCCs protected against the development of hyperalgesia. Thus, the identification of SOCCs opens the way for targeted treatment and the blockade of the SOCCs/p-CaMKII $\alpha$ pathway is a new therapeutic target. However, how SOCCs are activated after the administration of remifentanil is unclear. Further studies on the upstream mechanisms of SOCCs are encouraged to exam the relationship with RIPH.

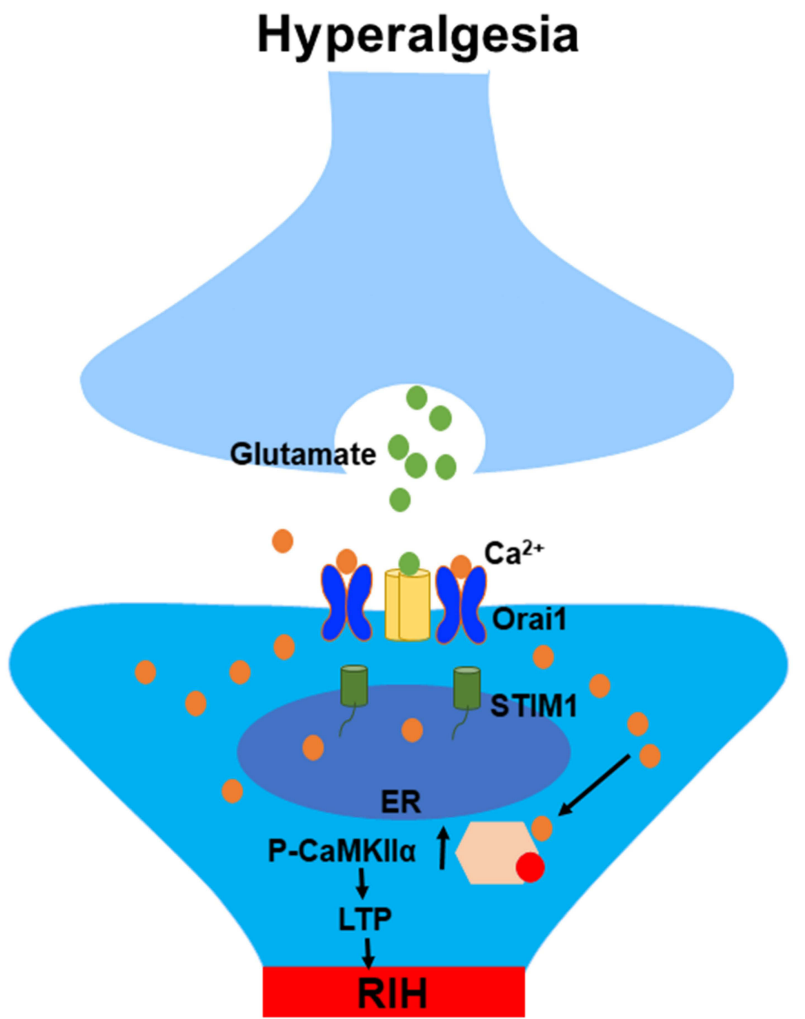

Figure 6 Probable signaling pathway for RIH.

Notes: The protein expression of STIMI and Orail are up-regulated after remifentanil infusion, which further enhances the store-operated Ca ${ }^{2+}$ entry (SOCE). Increased intracellular $\mathrm{Ca}^{2+}$ leads to the phosphorylation of CaMKIl $\alpha$. Moreover, CaMKIl $\alpha$ and its activation are parts of LTP hypersensitivity signaling which contributes to RIH. Abbreviations: SOCE, store-operated $\mathrm{Ca}^{2+}$ entry; LTP, long-term potentiation; RIH, remifentanil-induced hyperalgesia. 


\section{Abbreviations}

RIPH, remifentanil-induced postoperative hyperalgesia; SOCCs, store-operated calcium channels; STIM1, stromal interaction molecules 1; CaMKII $\alpha, \mathrm{Ca}^{2+} /$ calmodulindependent protein kinase II $\alpha$; PWTL, paw withdrawal thermal latency; PWMT, paw withdrawal mechanical threshold; $\mathrm{ER}$, endoplasmic reticulum; SOCE, store-operated $\mathrm{Ca}^{2+}$ entry; LTP, long-term potentiation; CNS, central nervous system; PD, Parkinson's disease; AD, Alzheimer's disease; SNI, spared nerve injury; PBS, phosphate buffer saline; PFA, paraformaldehyde; SEM, standard error of measurement; ANOVA, analysis of variance; OIH, opioid-induced hyperalgesia; RIH, remifentanil-induced hyperalgesia.

\section{Data Sharing Statement}

The datasets used during the present study are available from the corresponding author upon reasonable request.

\section{Author Contributions}

Zhenhui Zhou: This author helped design the study, conduct the study, analyze the data, and write the manuscript; Meng Mao: This author helped conduct the study, analyze the data, and prepare the manuscript; Xuechun Cai: This author helped analyze the data and prepare the manuscript; Wei Zhu: This author helped design the study, prepare the manuscript; Jie Sun: This author helped design the study, analyze the data, prepare the manuscript, and obtain the research fund. All authors made substantial contributions to conception and design, acquisition of data, or analysis and interpretation of data; took part in drafting the article or revising it critically for important intellectual content; agreed to submit to the current journal; gave final approval of the version to be published; and agree to be accountable for all aspects of the work.

\section{Funding}

This work was supported by the National Natural Science Foundation of China (No.82071196), the Jiangsu Commission of Health (No. z201949) and the Basic Research Grant of Southeast University.

\section{Disclosure}

The authors report no conflicts of interest in this work.

\section{References}

1. Yu EHY, Tran DHD, Lam SW, Irwin MG. Remifentanil tolerance and hyperalgesia: short-term gain, long-term pain? Anaesthesia. 2016;71 (11):1347-1362. doi:10.1111/anae.13602
2. Comelon M, Raeder J, Stubhaug A, Nielsen CS, Draegni T, Lenz H. Gradual withdrawal of remifentanil infusion may prevent opioid-induced hyperalgesia. Br J Anaesth. 2016;116(4):524-530. doi:10.1093/bja/aev547

3. Zhang L, Shu R, Zhao Q, Li Y, Yu Y, Wang G. Preoperative butorphanol and flurbiprofen axetil therapy attenuates remifentanil-induced hyperalgesia after laparoscopic gynaecological surgery: a randomized double-blind controlled trial. $\mathrm{Br} J$ Anaesth. 2016;117(4):504-511. doi:10.1093/bja/aew248

4. Zhang L, Guo S, Zhao Q, et al. Spinal protein kinase mzeta regulates alpha-amino-3-hydroxy-5-methyl-4-isoxazolepropionic acid receptor trafficking and dendritic spine plasticity via kalirin-7 in the pathogenesis of remifentanil-induced postincisional hyperalgesia in rats. Anesthesiology. 2018;129(1):173-186. doi:10.1097/ALN.0000000 000002190

5. Gao Y, Zhou S, Pan Y, Gu L, He Y, Sun J. Wnt3a inhibitor attenuates remifentanil-induced hyperalgesia via downregulating spinal NMDA receptor in rats. J Pain Res. 2020;13:1049-1058. doi:10.2147/JPR. S250663

6. Munoz F, Hu H. The role of store-operated calcium channels in pain. Adv Pharmacol. 2016;75:139.

7. Desvignes L, Weidinger C, Shaw P, et al. STIM1 controls T cellmediated immune regulation and inflammation in chronic infection. J Clin Invest. 2015;125(6):2347-2362. doi:10.1172/JCI80273

8. Wei F, Vadakkan KI, Toyoda H, et al. Calcium calmodulin-stimulated adenylyl cyclases contribute to activation of extracellular signal-regulated kinase in spinal dorsal horn neurons in adult rats and mice. $J$ Neurosci. 2006;26(3):851-861. doi:10.1523/ JNEUROSCI.3292-05.2006

9. Kuang X, Liu Y, Chang Y, et al. Inhibition of store-operated calcium entry by sub-lethal levels of proteasome inhibition is associated with STIM1/STIM2 degradation. Cell Calcium. 2016;59(4):172-180. doi:10.1016/j.ceca.2016.01.007

10. Ryazantseva M, Goncharova A, Skobeleva K, et al. Presenilin-1 delta E9 mutant induces STIM1-driven store-operated calcium channel hyperactivation in hippocampal neurons. Mol Neurobiol. 2018;55 (6):4667-4680. doi:10.1007/s12035-017-0674-4

11. Qi Z, Wang Y, Zhou H, et al. The central analgesic mechanism of YM-58483 in attenuating neuropathic pain in rats. Cell Mol Neurobiol. 2016;36(7):1035-1043. doi:10.1007/s10571-015-0292-5

12. Gao X, Xia J, Munoz FM, et al. STIMs and Orail regulate cytokine production in spinal astrocytes. J Neuroinflamm. 2016;13:1-13. doi:10.1186/s12974-016-0594-7

13. Gao R, Gao X, Xia J, et al. Potent analgesic effects of a store-operated calcium channel inhibitor. Pain. 2013;154 (10):2034-2044. doi:10.1016/j.pain.2013.06.017

14. Gao XH, Gao R, Tian YZ, et al. A store-operated calcium channel inhibitor attenuates collagen-induced arthritis. Brit J Pharmacol. 2015;172(12):2991-3002. doi:10.1111/bph.13104

15. Qi F, Liu T, Zhang X, et al. Ketamine reduces remifentanil-induced postoperative hyperalgesia mediated by CaMKII-NMDAR in the primary somatosensory cerebral cortex region in mice. Neuropharmacology. 2020;162:107783. doi:10.1016/j. neuropharm.2019.107783

16. Cui W, Wang S, Han R, Wang Q, Li J. CaMKII phosphorylation in primary somatosensory cortical neurons is involved in the inhibition of remifentanil-induced hyperalgesia by lidocaine in male Sprague-Dawley rats. J Neurosurg Anesthesiol. 2016;28(1):44-50. doi:10.1097/ANA.0000000000000177

17. Zhou Y, Liu D, Chen S, et al. Cellular and molecular mechanisms of calcium/calmodulin-dependent protein kinase II in chronic pain. $J$ Pharmacol Exp Ther. 2017;363(2):176-183. doi:10.1124/ jpet.117.243048

18. Herring BE, Nicoll RA. Long-term potentiation: from CaMKII to AMPA receptor trafficking. Annu Rev Physiol. 2016;78(1):351-365. doi:10.1146/annurev-physiol-021014-071753 
19. Cai Q, Zeng M, Wu X, et al. CaMKIIalpha-driven, phosphatase-checked postsynaptic plasticity via phase separation. Cell Res. 2021;31(1):37-51. doi:10.1038/s41422-020-00439-9

20. Wang Q, Zhao X, Li S, Han S, Peng Z, Li J. Phosphorylated CaMKII levels increase in rat central nervous system after large-dose intravenous remifentanil. Med Sci Monit Basic Res. 2013;19:118-125. doi:10.12659/MSMBR.883866

21. Ali ES, Rychkov GY, Barritt GJ. Metabolic disorders and cancer: hepatocyte store-operated $\mathrm{Ca}(2+)$ channels in nonalcoholic fatty liver disease. Adv Exp Med Biol. 2017;993:595-621.

22. Brennan TJ, Vandermeulen EP, Gebhart GF. Characterization of a rat model of incisional pain. Pain. 1996;64(3):493-501. doi:10.1016/ 0304-3959(95)01441-1

23. Mestre C, Pelissier T, Fialip J, Wilcox G, Eschalier A. A method to perform direct transcutaneous intrathecal injection in rats. J Pharmacol Toxicol Methods. 1994;32(4):197-200. doi:10.1016/ 1056-8719(94)90087-6

24. Chaplan SR, Bach FW, Pogrel JW, Chung JM, Yaksh TL. Quantitative assessment of tactile allodynia in the rat paw. J Neurosci Meth. 1994;53(1):55-63. doi:10.1016/0165-0270(94) 90144-9

25. Sun J, Chen SR, Chen H, Pan HL. $\mu$-Opioid receptors in primary sensory neurons are essential for opioid analgesic effect on acute and inflammatory pain and opioid-induced hyperalgesia. J Physiol. 2019;597(6):1661-1675. doi:10.1113/JP277428

26. Jensen TS, Finnerup NB. Allodynia and hyperalgesia in neuropathic pain: clinical manifestations and mechanisms. Lancet Neurol. 2014;13(9):924-935. doi:10.1016/S1474-4422(14)70102-4

27. Mercadante S, Arcuri E, Santoni A. Opioid-induced tolerance and hyperalgesia. CNS Drugs. 2019;33(10):943-955. doi:10.1007/ s40263-019-00660-0

28. Santonocito C, Noto A, Crimi C, Sanfilippo F. Remifentanil-induced postoperative hyperalgesia: current perspectives on mechanisms and therapeutic strategies. Local Reg Anesth. 2018;11:15-23. doi:10.2147/LRA.S143618

29. Martorano PP, Aloj F, Baietta S, et al. Sufentanil-propofol vs remifentanil-propofol during total intravenous anesthesia for neurosurgery. A multicentre study. Minerva Anestesiol. 2008;74 (6):233-243.

30. Roeckel L, Le Coz G, Gavériaux-Ruff C, Simonin F. Opioid-induced hyperalgesia: cellular and molecular mechanisms. Neuroscience. 2016;338:160-182.
31. Shu RC, Zhang LL, Wang CY, et al. Spinal peroxynitrite contributes to remifentanil-induced postoperative hyperalgesia via enhancement of divalent metal transporter 1 without iron-responsive element-mediated iron accumulation in rats. Anesthesiology. 2015;122(4):908-920. doi:10.1097/ALN.0000000000000562

32. Berna-Erro A, Braun A, Kraft R, et al. STIM2 regulates capacitive $\mathrm{Ca} 2+$ entry in neurons and plays a key role in hypoxic neuronal cell death. Sci Signal. 2009;2(93):a67. doi:10.1126/scisignal.2000522

33. Baba A, Yasui T, Fujisawa S, et al. Activity-evoked capacitative Ca2 + entry: implications in synaptic plasticity. J Neurosci. 2003;23 (21):7737-7741. doi:10.1523/JNEUROSCI.23-21-07737.2003

34. Stathopulos PB, Schindl R, Fahrner M, et al. STIM1/Orail coiled-coil interplay in the regulation of store-operated calcium entry. Nat Commun. 2013;4:2963. doi:10.1038/ncomms3963

35. Gemes G, Bangaru ML, Wu HE, et al. Store-operated $\mathrm{Ca} 2+$ entry in sensory neurons: functional role and the effect of painful nerve injury. $J \quad$ Neurosci. 2011;31(10):3536-3549. doi:10.1523/JNEUROSC I.5053-10.2011

36. Szteyn K, Gomez R, Berg KA, Jeske NA. Divergence in endothelin-1- and bradykinin-activated store-operated calcium entry in afferent sensory neurons. Asn Neuro. 2015;7:2. doi:10.1177/ 1759091415578714

37. Chen Y, Yang C, Wang ZJ. Ca2+/calmodulin-dependent protein kinase II is required for the initiation and maintenance of opioid-induced hyperalgesia. $J$ Neurosci. 2010;30(1):38-46. doi:10.1523/JNEUROSCI.4346-09.2010

38. Yuan Y, Sun Z, Chen Y, et al. Prevention of remifentanil induced postoperative hyperalgesia by dexmedetomidine via regulating the trafficking and function of spinal NMDA receptors as well as PKC and CaMKII level in vivo and in vitro. PLoS One. 2017;12(2): e171348.

39. Zhou J, Qi F, Hu Z, et al. Dezocine attenuates the remifentanilinduced postoperative hyperalgesia by inhibition of phosphorylation of CaMKIIa. Eur J Pharmacol. 2020;869:172882. doi:10.1016/j. ejphar.2019.172882

40. Voelkers M, Salz M, Herzog N, et al. Orai1 and Stim1 regulate normal and hypertrophic growth in cardiomyocytes. J Mol Cell Cardiol. 2010;48(6):1329-1334. doi:10.1016/j.yjmcc.2010.01.020
Journal of Pain Research

\section{Publish your work in this journal}

The Journal of Pain Research is an international, peer reviewed, open access, online journal that welcomes laboratory and clinical findings in the fields of pain research and the prevention and management of pain. Original research, reviews, symposium reports, hypothesis formation and commentaries are all considered for publication. The manuscript management system is completely online and includes a very quick and fair peer-review system, which is all easy to use. Visit http:// www.dovepress.com/testimonials.php to read real quotes from published authors. 